\title{
Blinding: an essential component in decreasing risk of bias in experimental designs
}

\section{Dorothy Forbes}

10.1136/eb-2013-101382

Correspondence to:

Dr Dorothy Forbes

Faculty of Nursing, University of

Alberta, Level 3, Edmonton

Clinic Health Academy (ECHA),

11405-87 Ave, Edmonton,

Alberta, Canada T6G 1C9;

dorothy.forbes@ualberta.ca

\section{What is blinding?}

Blinding (or masking) is the process used in experimental research by which study participants, persons caring for the participants, persons providing the intervention, data collectors and data analysts are kept unaware of group assignment (control vs intervention). Blinding aims to reduce the risk of bias that can be caused by an awareness of group assignment. With blinding, outcomes can be attributed to the intervention itself and not influenced by behaviour or assessment of outcomes that can result purely from knowledge of group allocation.

\section{Why incorporate blinding?}

Lack of blinding in randomised controlled trials (RCTs) has been shown to be associated with more exaggerated estimated intervention effects, by $9 \%$ on average. ${ }^{1}$ Studies with subjective outcomes are more likely to show these exaggerated estimates. ${ }^{2}$ In a systematic review of 250 RCTs, researchers observed a significant difference in the size of the estimated treatment effect between trials that reported 'double-blinding' compared with those that did not $(p=0.01)$, with an overall OR 17\% larger in studies that did not report blinding. ${ }^{3}$ In addition, studies are often simply described as 'blind' or 'double-blind' and do not specify who was blinded, ${ }^{4}$ which means blinding of one or more of the participants, healthcare providers, outcome assessors and analysts. Identification of who was blinded and how this was achieved would be valuable to assess potential for bias. ${ }^{5}$

Performance bias refers to systematic differences between the treatment and control groups resulting from care that was provided, or exposure to factors other than the interventions of interest. After enrolment into the study, blinding of participants and personnel may reduce the risk that knowledge of which intervention was received affects outcomes. If blinding is not incorporated or is unsuccessful, participants may respond better if they know they have received a promising new treatment. On the other hand, if participants are aware that they are not receiving an active treatment they may be less likely to comply with the study protocol, more likely to seek additional treatment and more likely to leave the study without providing outcome data. ${ }^{5}$ The healthcare providers who are blinded to participant allocation are much less likely to transfer their values to participants or to provide differential treatment to the active and placebo groups. ${ }^{5}$ However, blinding may not be possible in some studies where the intervention is obvious to the participants and/or persons administering the intervention (eg, an exercise intervention). Such studies can take other measures to reduce the risk of bias, such as treating participants according to a strict protocol to reduce the risk of differential behaviours by persons administering the intervention.
Blinding of outcome assessors is equally important to reduce the introduction of bias into the assessments and should be attempted whenever possible. ${ }^{5}$ Outcome assessments may be made by the participants themselves, by their healthcare providers, or by independent assessors. Blinding of the statistical analysts is achievable by simply labelling the participants' data with nonidentifying codes. ${ }^{5}$

\section{How to implement blinding?}

Blinding is not a simple procedure. The researchers often need to engage a variety of approaches to enhance blinding. Boutron et $a l^{6}$ conducted a systematic review of methods used in pharmacological RCTs to establish blinding of patients and/or healthcare providers. These included providing treatments in identical form, specific methods to mask characteristics of the treatments (eg, added flavour or colour), or use of double dummy procedures and even simulation of an injection.

Methods to avoid unblinding involved use of active placebo, centralised assessment of side effects, and patients informed only in part about the potential side effects of each treatment. Some of the methods used for blinding outcome assessors included centralised assessment of complementary investigations, clinical examination that involved the use of video, audiotape or photography, and adjudication of clinical events. Clearly there are ethical considerations to blinding. All blinding approaches should be explained as part of the method and receive ethical approval from research ethics boards.

\section{How to assess if blinding has been successful?}

An attempt to blind participants and personnel does not always ensure successful blinding in practice. For example, for many blinded drug trials, the side effects of the drugs can reveal group allocation, unless the study compares two rather similar interventions leg, drugs with similar side effects, or uses an active placebo. ${ }^{6}$ It has been suggested that it would be useful to ask trial participants at the end of the trial to guess which treatment they have received, ${ }^{7}{ }^{8}$ and some reviews of such reports have been published. ${ }^{79}$ Evidence of correct guesses exceeding 50\% would suggest that blinding may have been broken. However, responses may simply reflect the patients' experiences in the trial. A good outcome will tend to be more often attributed to an active treatment, and a poor outcome to a placebo. ${ }^{10}$

Risk of bias may be high for some outcomes and low for others. For example, knowledge of the assigned intervention may impact on behavioural outcomes (eg, number of visits to their physicians), while not impacting on physiological outcomes or mortality. Thus, assessments of risk of bias resulting from lack of blinding may need to be made separately for different 
outcomes. Rather than assessing risk of bias for each outcome separately, it is often convenient to group outcomes with similar risks of bias. For example, there may be a common assessment for all subjective outcomes (eg, quality of life) that is different from objective outcomes (eg, blood work). ${ }^{11}$

In summary, when considering the effectiveness of blinding in reducing the risk of bias, it is important to consider specifically:

1 Were the participants and study personnel blinded or not blinded?

2 Who assessed the outcomes and were they blinded or not blinded?

3 What was the risk of bias in the outcome assessment considering the subjectivity or objectivity of an outcome? ${ }^{11}$

\section{Competing interests None}

\section{References}

1. Pildal J, Hróbjartsson A, Jørgensen KJ, et al. Impact of allocation concealment on conclusions drawn from meta-analyses of randomized trials. Int J Epidemiol 2007;36:847-57.

2. Wood L, Egger M, Gluud LL, et al. Empirical evidence of bias in treatment effect estimates in controlled trials with different interventions and outcomes: meta-epidemiological study. $B M J$ 2008;336:601-5.

3. Schulz KF, Chalmers I, Hayes RJ, et al. Empirical evidence of bias. Dimensions of methodological quality associated with estimates of treatment effects in controlled trials. JAMA 1995;273:408-12.

4. Schulz KF, Chalmers I, Altman DG. The landscape and lexicon of blinding in randomized trials. Ann Intern Med 2002;136:254-9.

5. Karanicolas PJ, Farrokhyar F, Bhandari M. Blinding: Who, what, when, why, and how? Can J Surg 2010;53:345-8.

6. Boutron I, Estellat C, Guittet L, et al. Methods of blinding in reports of randomized controlled trials assessing pharmacologic treatments: a systematic review. PLoS Med 2006;3:1931-9.

7. Fergusson D, Glass KC, Waring D, et al. Turning a blind eye: the success of blinding reported in a random sample of randomised, placebo controlled trials. BMJ 2004;328:432.

8. Rees JR, Wade TJ, Levy DA, et al. Changes in beliefs identify unblinding in randomized controlled trials: a method to meet CONSORT guidelines. Contemp Clin Trials 2005;26:25-37.

9. Hróbjartsson A, Forfang E, Haahr MT, et al. Blinded trials taken to the test: an analysis of randomized clinical trials that report tests for the success of blinding. Int J Epidemiol 2007;36:654-63.

10. Sackett DL. Commentary: measuring the success of blinding in RCTs: don't, must, can't or needn't? Int J Epidemiol 2007;36:664-5.

11. Higgins JPT, Green S. Cochrane handbook for systematic reviews of interventions: version 5.0.1. 2011. http://www. cochrane-handbook.org 